\title{
The Existence and Uniqueness of Solutions for a Class of Nonlinear Fractional Differential Equations with Infinite Delay
}

\author{
Azizollah Babakhani, ${ }^{1}$ Dumitru Baleanu, ${ }^{2,3,4}$ and Ravi P. Agarwal, \\ ${ }^{1}$ Department of Mathematics, Faculty of Basic Science, Babol University of Technology, Babol 47148-71167, Iran \\ ${ }^{2}$ Department of Mathematics and Computer Science, Cankaya University, Turkey \\ ${ }^{3}$ Department of Chemical and Materials Engineering, Faculty of Engineering, King Abdulaziz University, \\ P.O. Box 80204, Jeddah 21589, Saudi Arabia \\ ${ }^{4}$ Institute of Space Sciences, P.O. Box MG-23, r 76900 Magurele-Bucharest, Romania \\ ${ }^{5}$ Department of Mathematics, Texas A \& M University-Kingsville, 700 University, Boulevard Kingsville, USA \\ ${ }^{6}$ Department of Mathematics, King Abdulaziz University, P.O. Box 80204, Jeddah 21589, Saudi Arabia
}

Correspondence should be addressed to Dumitru Baleanu; dumitru@cankaya.edu.tr

Received 28 October 2012; Accepted 2 February 2013

Academic Editor: Bashir Ahmad

Copyright (C) 2013 Azizollah Babakhani et al. This is an open access article distributed under the Creative Commons Attribution License, which permits unrestricted use, distribution, and reproduction in any medium, provided the original work is properly cited.

We prove the existence and uniqueness of solutions for two classes of infinite delay nonlinear fractional order differential equations involving Riemann-Liouville fractional derivatives. The analysis is based on the alternative of the Leray-Schauder fixed-point theorem, the Banach fixed-point theorem, and the Arzela-Ascoli theorem in $\Omega=\left\{y:(-\infty, b] \rightarrow \mathbb{R}:\left.y\right|_{(-\infty, 0]} \in \mathscr{B}\right\}$ such that $\left.y\right|_{[0, b]}$ is continuous and $\mathscr{B}$ is a phase space.

\section{Introduction}

Fractional derivatives and integrals have been vastly used in different fields, facing a huge development especially during the last few decades (see, e.g., [1-9] and the references therein). The approaches based on fractional calculus establish models of engineering systems better than the ordinary derivatives approaches [1-6].

In particular, fractional differential equations as an important research branch of fractional calculus attracted much more attention (see, e.g., [10-20] and the references therein). Also varieties of schemes for numerical solutions of fractional differential equations are reported (see, e.g., $[6,21-23]$ and the references therein). We notice that some investigations have been done on the existence and uniqueness of solutions for fractional differential equations with delay (see, e.g., [24, 25] and the references therein).
Having all the aforementioned facts in mind, in this paper we study the existence and uniqueness of solutions for a class of delayed fractional differential equations, namely,

$$
\begin{gathered}
\mathscr{L}(\mathscr{D}) y(t)=f\left(t, y_{t}\right), \quad t \in J=[0, b], \\
y(t)=\phi(t), \quad t \in(-\infty, 0],
\end{gathered}
$$

where $\mathscr{L}(\mathscr{D})=D_{0^{+}}^{\alpha}-t^{n} D_{0^{+}}^{\beta}, 0<\beta<\alpha<1, n$ is a positive integer, $f: J \times \mathscr{B} \rightarrow \mathbb{R}$ is a given function satisfying some assumptions that will be specified later, $\phi \in \mathscr{B}$ with $\phi(0)=$ 0 , and $\mathscr{B}$ is called a phase space that will be defined later. $D_{0^{+}}^{\alpha}$ and $D_{0^{+}}^{\beta}$ are the standard Riemann-Liouville fractional derivatives. $y_{t}$, which is an element $\mathscr{B}$, is defined as any function $y$ on $(-\infty, b]$ as follows:

$$
y_{t}(s)=y(t+s), \quad s \in(-\infty, 0], t \in J .
$$


Here $y_{t}(\cdot)$ represents the preoperational state from time $-\infty$ up to time $t$. We also consider the following nonlinear fractional differential equation:

$$
\begin{gathered}
\mathscr{L}(\mathscr{D})\left\{y(t)-g\left(t, y_{t}\right)\right\}=f\left(t, y_{t}\right), \quad t \in J, \\
y(t)=\phi(t), \quad t \in(-\infty, 0]
\end{gathered}
$$

where $\alpha, \beta, f, \phi$, and $\mathscr{L}(\mathscr{D})$ are as (1) and $g: J \times \mathscr{B} \rightarrow \mathbb{R}$ is a given function which satisfies $g(0, \phi)=0$.

The notion of the phase space $\mathscr{B}$ plays an important role in the study of both qualitative and quantitative theories for functional differential equations. A common choice is a seminormed space satisfying suitable axioms, which was introduced by Hale and Kato [26].

Our approach is based on the Banach fixed-point theorem and on the nonlinear alternative of Leray-Schauder type [27, 28]. The organization of the paper is as follows.

In Section 2, we present some basic mathematical tools used in the paper. The main results are presented in Section 3. Section 4 is dedicated to our conclusions.

\section{Preliminaries}

In this section, we present some basic notations and properties which are used throughout this paper. First of all, we will explain the phase space $\mathscr{B}$ introduced by Hale and Kato [26]. Let $\mathbb{R}^{\leq 0}=(-\infty, 0], \mathbb{R}^{\geq 0}=[0,+\infty), \mathbb{R}=(-\infty,+\infty)$, and let $E$ be a Banach space with norm $|\cdot|_{E}$. Further, let $\mathscr{B}$ be a linear space of functions mapping $\mathbb{R}^{-}$into $E$ with seminorm $|\cdot|_{\mathscr{B}}$ having the following axioms,

$\left(\mathrm{B}_{1}\right)$ If $y:(-\infty, \sigma+b) \rightarrow E, b>0$ is continuous on $[\sigma, \sigma+$ $b)$ and $y_{\sigma} \in \mathscr{B}$, then $y_{t} \in \mathscr{B}$ and $y_{t}$ are continuous for any $t \in[\sigma, \sigma+b)$.

$\left(\mathrm{B}_{2}\right)$ There exist functions $k(t)>0$ and $m(t) \geq 0$ with the following properties. (i) $k(t)$ is continuous for $t \in$ $\mathbb{R}^{\geq 0}$. (ii) $m(t)$ is locally bounded for $t \in \mathbb{R}^{\geq 0}$. (iii) For every function, $y$ has the properties of $\left(B_{1}\right)$ and $t \in[\sigma, \sigma+b)$, holds that $\left|y_{t}\right|_{\mathscr{B}} \leq k(t-\sigma) \sup \left\{|y(s)|_{E}\right.$ : $\sigma \leq s \leq t\}+m(t-\sigma)\left|y_{\sigma}\right|_{\mathscr{B}}$.

$\left(\mathrm{B}_{3}\right)$ There exists a positive constant $L$ such that $|\phi(0)|_{E} \leq$ $L|\phi|_{\mathscr{B}}$ for all $\phi \in \mathscr{B}$.

$\left(\mathrm{B}_{4}\right)$ The quotient space $\widehat{\mathscr{B}}:=\mathscr{B} /|\cdot|_{\mathscr{B}}$ is a Banach space.

We notice that in this paper, we select $\sigma=0$ and $E=\mathbb{R}$; thus (iii) can be converted to $\left|y_{t}\right|_{\mathscr{B}} \leq k(t) \sup \left\{|y(s)|_{E}: 0 \leq s \leq\right.$ $t\}+m(t)\left|y_{0}\right|_{\mathscr{B}}$, for all $t \in[0, b)$.

See [28] for examples of the phase space $\mathscr{B}$ satisfying all axioms $\left(\mathrm{B}_{1}\right)-\left(\mathrm{B}_{4}\right)$.

Let $\mathbb{R}^{+}=(0,+\infty)$ and $C^{0}\left(\mathbb{R}^{+}\right)$be the space of all continuous real function on $\mathbb{R}^{+}$. Consider also the space $C^{0}(\mathbb{R})^{\geq 0}$ of all continuous real functions on $\mathbb{R}^{\geq 0}$ which later identifies with the class of all $f \in C^{0}\left(\mathbb{R}^{+}\right)$such that $\lim _{t \rightarrow 0^{+}} f(t)=f\left(0^{+}\right) \in \mathbb{R}$. By $C(J, \mathbb{R})$, we denote the Banach space of all continuous functions from $J$ into $\mathbb{R}$ with the norm $\|y\|_{\infty}:=\sup \{|y(t)|: t \in J\}$, where $|\cdot|$ is a suitable complete norm on $\mathbb{R}$.
The most common notation for $\alpha$ th order derivative of a real-valued function $y(t)$, which is defined in an interval denoted by $(a, b)$, is $D_{a}^{\alpha} y(t)$. Here, the negative value of $\alpha$ corresponds to the fractional integral.

Definition 1. For a function $y$ defined on an interval $[a, b]$, the Riemann-Liouville fractional integral of $y$ of order $\alpha>0$ is defined by $[1,6]$

$$
I_{a^{+}}^{\alpha} y(t)=\frac{1}{\Gamma(\alpha)} \int_{a}^{t}(t-s)^{\alpha-1} y(s) d s, \quad t>a,
$$

and the Riemann-Liouville fractional derivative of $y(t)$ of order $\alpha>0$ reads as

$$
D_{a^{+}}^{\alpha} y(t)=\frac{d^{n}}{d t^{n}}\left\{I_{a^{+}}^{n-\alpha} y(t)\right\}, \quad n-1<\alpha \leq n .
$$

Also, we denote $D_{a^{+}}^{\alpha} y(t)$ as $D_{a}^{\alpha} y(t)$ and $I_{a^{+}}^{\alpha} y(t)$ as $I_{a}^{\alpha} y(t)$. Further, $D_{0^{+}}^{\alpha} y(t)$ and $I_{0^{+}}^{\alpha} y(t)$ are referred to as $D^{\alpha} y(t)$ and $I^{\alpha} y(t)$, respectively. If the fractional derivative $D_{a}^{\alpha} y(t)$ is integrable, then we have [4, page 71]

$$
\begin{aligned}
& I_{a}^{\alpha}\left(D_{a}^{\beta} y(t)\right) \\
&=I_{a}^{\alpha-\beta} y(t)-\left[I_{a}^{1-\beta} y(t)\right]_{t=a} \frac{(t-a)^{\alpha-1}}{\Gamma(\alpha)}, \\
& 0<\beta \leq \alpha<1 .
\end{aligned}
$$

If $y$ is continuous on $[a, b]$, then $D_{a}^{\alpha} y(t)$ is integrable, $\left.I^{1-\beta} y(t)\right|_{t=a}=0$, and

$$
I_{a}^{\alpha}\left(D_{a}^{\beta} y(t)\right)=I_{a}^{\alpha-\beta} y(t), \quad 0<\beta \leq \alpha<1 .
$$

Proposition 2. Let $y$ be continuous on $[0, b]$ and $n$ a nonnegative integer, then

$$
\text { (i) } \begin{aligned}
I^{\alpha}\left(t^{n} y(t)\right) & =\sum_{k=0}^{n}\left(\begin{array}{c}
-\alpha \\
k
\end{array}\right)\left[D^{k} t^{n}\right]\left[I^{\alpha+k} y(t)\right] \\
& =\sum_{k=0}^{n}\left(\begin{array}{c}
-\alpha \\
k
\end{array}\right) \frac{n ! t^{n-k}}{(n-k) !} I^{\alpha+k} y(t),
\end{aligned}
$$

(ii) $I^{\alpha}\left(t^{n} D^{\beta} y(t)\right)=\sum_{k=0}^{n}\left(\begin{array}{c}-\alpha \\ k\end{array}\right) \frac{n ! t^{n-k}}{(n-k) !} I^{\alpha-\beta+k} y(t)$,

where

$$
\begin{aligned}
\left(\begin{array}{c}
-\alpha \\
k
\end{array}\right) & =(-1)^{k} \frac{\Gamma(\alpha+1)}{k ! \Gamma(\alpha)}=(-1)^{k}\left(\begin{array}{c}
\alpha+k-1 \\
k
\end{array}\right) \\
& =\frac{\Gamma(1-\alpha)}{\Gamma(k+1) \Gamma(1-\alpha-k)} .
\end{aligned}
$$

Proof. (i) can be found in [6, page 53], and (ii) is an immediate consequence of (7), and (i).

Lemma 3 (see [29]). Let $v:[0, b] \rightarrow[0, \infty)$ be a real function and $w(\cdot)$ a nonnegative, locally integrable function on 
$[0, b]$. If there exist positive constants $a$ and $\alpha \in(0,1)$ such that $v(t) \leq w(t)+a \int_{0}^{t}(t-s)^{-\alpha} v(s) d s$, then there exists a constant $K=K(\alpha)$ such that $v(t) \leq w(t)+K a \int_{0}^{t} w(s)(t-s)^{-\alpha} d s$, for all $t \in[0, b]$.

In this paper we use the alternative Leray-Schauder's theorem and Banach's contraction principle for getting the main results. These theorems can be found in $[27,28]$.

\section{Existence and Uniqueness}

In this section, we prove the existence results for (1) and (3) by using the alternative of Leray-Schauder's theorem. Further, our results for the unique solution is based on the Banach contraction principle. Let us start by defining what we mean by a solution of (1). Let the space

$$
\begin{gathered}
\Omega=\left\{y:(-\infty, b] \longrightarrow \mathbb{R}:\left.y\right|_{(-\infty, 0]} \in \mathscr{B}\right. \\
\text { and } \left.\left.y\right|_{[0, b]} \text { is continuous }\right\} .
\end{gathered}
$$

A function $y \in \Omega$ is said to be a solution of (1) if $y$ satisfies (1). lemma.

For the existence results on (1), we need the following

Lemma 4. Equation (1) is equivalent to the Volterra integral equation

$$
y(t)=\sum_{k=0}^{n}\left(\begin{array}{c}
-\alpha \\
k
\end{array}\right) \frac{n ! t^{n-k}}{(n-k) !} I^{\alpha-\beta+k} y(t)+I^{\alpha} f\left(t, y_{t}\right), \quad t \in J .
$$

Proof. The proof is an immediate consequence of Proposition 2.

To study the existence and uniqueness of solutions for (1), we transform (1) into a fixed-point problem. Consider the operator $P: \Omega \rightarrow \Omega$ defined by

$$
P y(t)= \begin{cases}\mathscr{L}(I) y(t)+I^{\alpha} f\left(t, y_{t}\right), & t \in[0, b], \\ \phi(t), & t \in(-\infty, 0],\end{cases}
$$

where,

$$
\mathscr{L}(I)=\sum_{k=0}^{n}\left(\begin{array}{c}
-\alpha \\
k
\end{array}\right) \frac{n ! t^{n-k}}{(n-k) !} I^{\alpha-\beta+k} .
$$

Let $x(\cdot):(-\infty, b] \rightarrow \mathbb{R}$ be the function defined as

$$
x(t)= \begin{cases}0, & \text { if } t \in[0, b], \\ \phi(t), & \text { if } t \in(-\infty, 0] .\end{cases}
$$

Then, we get $x_{0}=\phi$. For each $z \in C([0, b], \mathbb{R})$ with $z(0)=0$, we denote by $\bar{z}$ the function defined as follows:

$$
\bar{z}(t)= \begin{cases}z(t), & \text { if } t \in[0, b] \\ 0, & \text { if } t \in(-\infty, 0]\end{cases}
$$

If $y(\cdot)$ satisfies the integral equation $y(t)=\mathscr{L}(I) y(t)+$ $I^{\alpha} f\left(t, y_{t}\right)$, then we can decompose $y(\cdot)$ as $y(t)=\bar{z}(t)+x(t)$, $-\infty<t \leq b$, which implies $y_{t}=\bar{z}_{t}+x_{t}$ for every $0 \leq t \leq b$, and the function $z(\cdot)$ satisfies

$$
z(t)=\mathscr{L}(I) z(t)+I^{\alpha} f\left(t, \bar{z}_{t}+x_{t}\right),
$$

set $C_{0}=\{z \in C([0, b], \mathbb{R}): z(0)=0\}$, and let $\|\cdot\|_{b}$ be the seminorm in $C_{0}$ defined by $\|z\|_{b}=\left\|z_{0}\right\|_{\mathscr{B}}+\sup \{|z(t)|: 0 \leq$ $t \leq b\}=\sup \{|z(t)|: 0 \leq t \leq b\}, z \in C_{0} . C_{0}$ is a Banach space with norm $\|\cdot\|_{b}$. Let the operator $F: C_{0} \rightarrow C_{0}$ be defined by

$$
F z(t)=\mathscr{L}(I) z(t)+I^{\alpha} f\left(t, \bar{z}_{t}+x_{t}\right)
$$

where $t \in[0, b]$. The operator $P$ has a fixed point equivalent to $F$ that has a fixed point too.

Theorem 5. Assume that $f$ is a continuous function, and there exist $p, q \in C\left(J, \mathbb{R}^{+}\right)$such that $|f(t, u)| \leq p(t)+q(t)\|u\|_{\mathscr{B}}, t \in$ $J, u \in \mathscr{B}$. Then, (1) has at least one solution on $(-\infty, b]$.

Proof. It is enough to show that the operator $F: C_{0} \rightarrow C_{0}$ defined as (18) satisfies the following: (i) $F$ is continuous, (ii) $F$ maps bounded sets into bounded sets in $C_{0}$, (iii) $F$ maps bounded sets into equicontinuous sets of $C_{0}$, and (iv) $F$ is completely continuous.

(i) Let $\left\{z_{n}\right\}$ converges to $z$ in $C_{0}$, then

$$
\begin{aligned}
& \left\|F z_{n}(t)-F z(t)\right\| \\
& \leq \sum_{k=0}^{n} \frac{\left|\left(\begin{array}{c}
-\alpha \\
k
\end{array}\right)\right| n ! t^{n-k}}{(n-k) !} I^{\alpha-\beta+k}\left|z_{n}(t)-z(t)\right| \\
& \quad+I^{\alpha}\left|f\left(t,\left(\bar{z}_{n}\right)_{t}+x_{t}\right)-f\left(t, \bar{z}_{t}+x_{t}\right)\right| \\
& \leq \sum_{k=0}^{n} \frac{\left|\left(\begin{array}{c}
-\alpha \\
k
\end{array}\right)\right| n ! b^{n-k}\left\|z_{n}-z\right\|}{(n-k) ! \Gamma(\alpha-\beta+k+1)} \\
& \quad+\frac{b^{\alpha}\left\|f\left(t,\left(\bar{z}_{n}\right)_{t}+x_{t}\right)-f\left(t, \bar{z}_{t}+x_{t}\right)\right\|}{\Gamma(\alpha+1)} .
\end{aligned}
$$

Hence, $\left\|F z_{n}(t)-F z(t)\right\| \rightarrow 0$ as $z_{n} \rightarrow z$, and thus $f$ is continuous.

(ii) For any $\lambda>0$, let $\mathscr{B}_{\lambda}=\left\{z \in C_{0}:\|z\|_{b} \leq \lambda\right\}$ be a bounded set. We show that there exists a positive 
constant $\mu$ such that $\|F z\|_{\infty} \leq \mu$. Let $z \in \mathscr{B}_{\lambda}$, since $f$ is a continuous function, we have for each $t \in[0, b]$,

$$
\begin{aligned}
& |F z(t)| \leq \sum_{k=0}^{n} \frac{\left|\left(\begin{array}{c}
-\alpha \\
k
\end{array}\right)\right| n ! t^{n-k}}{(n-k) ! \Gamma(\alpha-\beta+k)} \\
& \times \int_{0}^{b}(t-s)^{\alpha-\beta+k-1} z(s) \\
& +\frac{1}{\Gamma(\alpha)} \int_{0}^{t}(t-s)^{\alpha-1} f\left(s, \bar{z}_{s}+x_{s}\right) d s \\
& \leq \sum_{k=0}^{n} \frac{\left|\left(\begin{array}{c}
-\alpha \\
k
\end{array}\right)\right| n ! b^{n+\alpha-\beta}}{(n-k) ! \Gamma(\alpha-\beta+k+1)}\|z\|_{b}+\frac{1}{\Gamma(\alpha)} \\
& \times \int_{0}^{t}(t-s)^{\alpha-1}\left[p(s)+q(s)\left\|\bar{z}_{s}+x_{s}\right\|_{\mathscr{B}}\right] d s \\
& \leq \sum_{k=0}^{n} \frac{\left|\left(\begin{array}{c}
-\alpha \\
k
\end{array}\right)\right| n ! b^{n+\alpha-\beta}}{(n-k) ! \Gamma(\alpha-\beta+k+1)}\|z\|_{b} \\
& +\frac{b^{\alpha}\|p\|_{\infty}}{\Gamma(\alpha+1)}+\frac{b^{\alpha}\|q\|_{\infty}}{\Gamma(\alpha+1)}\left\{\left\|\bar{z}_{s}\right\|_{\mathscr{B}}+\left\|x_{s}\right\|_{\mathscr{B}}\right\} \\
& \leq \sum_{k=0}^{n} \frac{\left|\left(\begin{array}{c}
-\alpha \\
k
\end{array}\right)\right| n ! b^{n+\alpha-\beta}}{(n-k) ! \Gamma(\alpha-\beta+k+1)}\|z\|_{b} \\
& +\frac{b^{\alpha}\|p\|_{\infty}}{\Gamma(\alpha+1)}+k_{b} \lambda+m_{b}\|\phi\|_{\mathscr{B}}:=\mu,
\end{aligned}
$$

where $m_{b}=\sup \{|m(t)|: t \in[0, b]\}$, and $k_{b}=$ $\sup \{|k(t)|: t \in[0, b]\}$. Hence, we obtain $\|F z\|_{\infty} \leq \mu$.

(iii) Let $t_{1}, t_{2} \in[0, b]$ and $t_{1}<t_{2}$. Let $\mathscr{B}_{\lambda}$ be a bounded set of $C_{0}$ as in (ii) and $z \in \mathscr{B}_{\lambda}$, then given $\epsilon>0$ choose

$$
\begin{array}{r}
\delta=\min \left\{\frac{1}{2 \Lambda_{1}} \epsilon^{1 / \alpha}, \frac{1}{2(n+1) \Lambda_{2}} \epsilon^{1 /(\alpha-\beta+k)}:\right. \\
k=0,1, \ldots, n\},
\end{array}
$$

where

$$
\begin{gathered}
\Lambda_{1}=2 \frac{\|p\|_{\infty}+\Lambda\|q\|_{\infty}}{\Gamma(\alpha+1)}, \\
\Lambda_{2}=\sum_{k=0}^{n} \frac{2\left|\left(\begin{array}{c}
-\alpha_{n} \\
k
\end{array}\right)\right| k ! b^{n-k}\|z\|_{b}}{(n-k) ! \Gamma(\alpha-\beta+k+1)},
\end{gathered}
$$

and $\Lambda=k_{b} \lambda+m_{b}\|\phi\|_{\mathscr{B}}$. If $\left|t_{2}-t_{1}\right|<\delta$, then

$$
\begin{aligned}
& \left|F z\left(t_{2}\right)-F z\left(t_{1}\right)\right| \\
& \leq \sum_{k=0}^{n} \frac{\left|\left(\begin{array}{c}
-\alpha_{n} \\
k
\end{array}\right)\right| k ! b^{n-k}}{(n-k) ! \Gamma(\alpha-\beta+k)}\|z\|_{b} \\
& \times \mid \int_{0}^{t_{1}}\left\{\left(t_{2}-s\right)^{\alpha-\beta+k-1}-\left(t_{1}-s\right)^{\alpha-\beta+k-1}\right\} d s \\
& +\int_{t_{1}}^{t_{2}}\left(t_{2}-s\right)^{\alpha-\beta+k-1} d s \\
& +\frac{1}{\Gamma(\alpha)} \mid \int_{0}^{t_{1}}\left\{\left(t_{2}-s\right)^{\alpha-1}-\left(t_{1}-s\right)^{\alpha-1}\right\} f\left(s, \bar{z}_{s}+x_{x}\right) d s \\
& +\int_{t_{1}}^{t_{2}}\left(t_{2}-s\right)^{\alpha-1} f\left(s, \bar{z}_{s}+x_{x}\right) d s \\
& \leq \sum_{k=0}^{n} \frac{2\left|\left(\begin{array}{c}
-\alpha_{n} \\
k
\end{array}\right)\right| k ! b^{n-k}}{(n-k) ! \Gamma(\alpha-\beta+k+1)}\|z\|_{b}\left(t_{2}-t_{1}\right)^{\alpha-\beta+k} \\
& +\frac{\|p\|_{\infty}+\Lambda\|q\|_{\infty}}{\Gamma(\alpha+1)}\left\{\int_{0}^{t_{1}}\left\{\left(t_{2}-s\right)^{\alpha-1}-\left(t_{1}-s\right)^{\alpha-1}\right\} d s\right. \\
& \left.+\int_{t_{1}}^{t_{2}}\left(t_{2}-s\right)^{\alpha-1} d s\right\} \\
& \leq \sum_{k=0}^{n} \frac{2\left|\left(\begin{array}{c}
-\alpha_{n} \\
k
\end{array}\right)\right| k ! b^{n-k}}{(n-k) ! \Gamma(\alpha-\beta+k+1)}\|z\|_{b}\left(t_{2}-t_{1}\right)^{\alpha-\beta+k} \\
& +2 \frac{\|p\|_{\infty}+\Lambda\|q\|_{\infty}}{\Gamma(\alpha+1)}\left(t_{2}-t_{1}\right)^{\alpha} \\
& =\Lambda_{2} \delta^{\alpha-\beta+k}+\Lambda_{1} \delta^{\alpha}<\frac{\epsilon}{2}+\frac{\epsilon}{2}=\epsilon,
\end{aligned}
$$

where $\left\|\bar{z}_{s}+x_{s}\right\|_{\mathscr{B}} \leq\left\|\bar{z}_{s}\right\|_{\mathscr{B}}+\left\|x_{s}\right\|_{\mathscr{B}} \leq k_{b} \lambda+m_{b}\|\phi\|_{\mathscr{B}}:=$ $\Lambda$. Hence, $F\left(\mathscr{B}_{\lambda}\right)$ is equicontinuous.

(iv) It is an immediate consequence from (i)-(iii), together with the Arzela-Ascoli theorem.

We show in the following that there exists an open set $U \subseteq C_{0}$ with $z \neq \gamma F(z)$ for $\gamma \in(0,1)$ and $z \in \partial U$. Let $z \in C_{0}$ and $z=\gamma F(z)$ for some $0<\gamma<1$. Then, for each $t \in[0, b]$, we have $z(t)=\lambda\left\{\mathscr{L}(I) z(t)+I^{\alpha} f\left(t, \bar{z}_{t}+x_{t}\right)\right\}$. It follows by assumption of the theorem

$$
\begin{aligned}
|z(t)| \leq & \sum_{k=0}^{n} \frac{\left|\left(\begin{array}{c}
-\alpha_{n} \\
k
\end{array}\right)\right| k ! b^{n-k}}{(n-k) ! \Gamma(\alpha-\beta+k)} \int_{0}^{t}(t-s)^{\alpha-\beta+k-1}|z(s)| d s \\
& +\frac{1}{\Gamma(\alpha)} \int_{0}^{t}(t-s)^{\alpha-1}\left|f\left(s, \bar{z}_{s}+x_{x}\right)\right| d s
\end{aligned}
$$




$$
\begin{aligned}
\leq & \sum_{k=0}^{n} \frac{\left|\left(\begin{array}{c}
-\alpha_{n} \\
k
\end{array}\right)\right| k ! b^{n-k}\|z\|_{b}}{(n-k) ! \Gamma(\alpha-\beta+k+1)} \\
& +\frac{1}{\Gamma(\alpha)} \int_{0}^{t}(t-s)^{\alpha-1} q(s)\left\|\bar{z}_{s}+x_{s}\right\|_{\mathscr{B}} d s \\
& +\frac{b^{\alpha}\|p\|_{\infty}}{\Gamma(\alpha+1)}
\end{aligned}
$$

On other hand, we have

$$
\begin{aligned}
\left\|\bar{z}_{s}+x_{s}\right\|_{B} \leq & \left\|\bar{z}_{s}\right\|_{\mathscr{B}}+\left\|x_{s}\right\|_{\mathscr{B}} \\
\leq & k(t) \sup \{|z(s)|: 0 \leq s \leq t\} \\
& +m(t)\left\|z_{0}\right\|_{\mathscr{B}} \\
& +k(t) \sup \{|x(s)|: 0 \leq s \leq t\} \\
& +m(t)\left\|x_{0}\right\|_{\mathscr{B}} \\
\leq & k_{b} \sup \{|z(s)|: 0 \leq t \leq t\} \\
& +m_{b}\|\phi\|_{\mathscr{B}} .
\end{aligned}
$$

If we let $\delta(t)$ the right-hand side of (25), then $\left\|\bar{z}_{s}+x_{s}\right\|_{\mathscr{B}} \leq \delta(t)$ and, therefore,

$$
\begin{aligned}
|z(t)| \leq & \sum_{k=0}^{n} \frac{\left|\left(\begin{array}{c}
-\alpha_{n} \\
k
\end{array}\right)\right| k ! b^{n-k}\|z\|_{b}}{(n-k) ! \Gamma(\alpha-\beta+k+1)} \\
& +\frac{1}{\Gamma(\alpha)} \int_{0}^{t}(t-s)^{\alpha-1} q(s) \delta(s) d s+\frac{b^{\alpha}\|p\|_{\infty}}{\Gamma(\alpha+1)} .
\end{aligned}
$$

Using the aforementioned inequality and the definition of $\delta$, we get

$$
\begin{aligned}
\delta(t) \leq \sum_{k=0}^{n} & \frac{\left|\left(\begin{array}{c}
-\alpha_{n} \\
k
\end{array}\right)\right| k ! b^{n-k}\|z\|_{b} k_{b}}{(n-k) ! \Gamma(\alpha-\beta+k+1)}+m_{b}\|\phi\|_{\mathscr{B}} \\
+ & \frac{k_{b} b^{\alpha}\|p\|_{\infty}}{\Gamma(\alpha+1)}+\frac{k_{b}\|q\|_{\infty}}{\Gamma(\alpha)} \\
& \times \int_{0}^{t}(t-s)^{\alpha-1} \delta(s) d s .
\end{aligned}
$$

Then, using Lemma 3, there exists a constant $\Delta$ such that

$$
\begin{aligned}
|\delta(t)| \leq & \frac{1}{2} k_{b} \Lambda_{2}+m_{b}\|\phi\|_{\mathscr{B}} \\
& +\frac{k_{b} b^{\alpha}\|p\|_{\infty}}{\Gamma(\alpha+1)}+\Delta \frac{k_{b}\|q\|_{\infty}}{\Gamma(\alpha)} \int_{0}^{t}(t-s)^{\alpha-1} R d s
\end{aligned}
$$

where $\Lambda_{2}$ is mentioned in (22), and

$$
R=\frac{1}{2} k_{b} \Lambda_{2}+m_{b}\|\phi\|_{\mathscr{B}}+\frac{k_{b} b^{\alpha}\|p\|_{\infty}}{\Gamma(\alpha+1)} .
$$

Hence,

$$
\|\delta\|_{\infty} \leq R+\frac{R \Delta b^{\alpha} k_{b}\|q\|_{\infty}}{\Gamma(\alpha+1)}:=\widetilde{M},
$$

and then $\|z\|_{\infty} \leq \Lambda_{2}+\widetilde{M}\left\|I^{\alpha} q\right\|_{\infty}+b^{\alpha}\|p\|_{\infty} / \Gamma(\alpha+1)$. Therefore,

$$
\|z\|_{\infty} \leq \frac{\widetilde{M}\left\|I^{\alpha} q\right\|_{\infty}+b^{\alpha}\|p\|_{\infty} / \Gamma(\alpha+1)}{1-\Lambda_{2}}:=\Delta^{*} .
$$

Set $U=\left\{z \in C_{0}:\|z\|_{b}<\Delta^{*}+1\right\}$. Then, $F: \bar{U} \rightarrow C_{0}$ is continuous and completely continuous. From the choice of $U$, there is no $z \in \partial U$ such that $z=\gamma F(z)$, for $\gamma \in(0,1)$; therefore, by the nonlinear alternative of the Leray-Schauder theorem, the proof is complete.

Theorem 6. Let $f: J \times B \rightarrow \mathbb{R}$ be a continuous function. If there exists a positive constant $l$ such that $|f(t, u)-f(t, v)| \leq$ $l\|u-v\|_{\mathscr{B}}, t \in J, u, v \in \mathscr{B}$, and $0<T+l k_{b} b^{\alpha} / \Gamma(\alpha+1):=L<1$ then (1) has a unique solution in the interval $(-\infty, b]$, where,

$$
T=\sum_{k=0}^{n} \frac{\left|\left(\begin{array}{c}
-\alpha_{n} \\
k
\end{array}\right)\right| k ! b^{n-k}}{(n-k) ! \Gamma(\alpha-\beta+k+1)} .
$$

Proof. The solution of (1) is equivalent to the solution of the integral equation (17). Hence, it is enough to show that the operator $F: C_{0} \rightarrow C_{0}$, satisfies the Banach fixed-point theorem. Consider $u, v \in C_{0}$ and for each $t \in[0, b]$, we have

$$
\begin{aligned}
& |F(z)(t)-F(u)(t)| \\
& \quad \leq T\|u-v\|_{b}+\frac{1}{\Gamma(\alpha)} \int_{0}^{t}(t-s)^{\alpha-1} l\left\|\bar{u}_{s}-\bar{v}_{s}\right\|_{\mathscr{B}} d s \\
& \quad \leq T\|u-v\|_{b}+\frac{l}{\Gamma(\alpha)} \int_{0}^{t}(t-s)^{\alpha-1}\left\|\bar{u}_{s}-\bar{v}_{s}\right\|_{\mathscr{B}} d s \\
& \quad \leq T\|u-v\|_{b}+\frac{l}{\Gamma(\alpha)} \int_{0}^{t}(t-s)^{\alpha-1} \\
& \quad \leq\left\{T+\frac{l k_{b}}{\Gamma(\alpha)} \int_{0}^{t}(t-s)^{\alpha-1} l d s\right\}|u-v|_{b} \\
& \quad \leq\left\{T+\frac{l k_{b} b^{\alpha}}{\Gamma(\alpha+1)}\right\}\|u-v\|_{b}=L\|u-v\|_{b} .
\end{aligned}
$$

Hence, $\|F(z)-F(v)\|_{b} \leq L\left\|z-z^{*}\right\|_{b}$, and then $F$ is a contraction. Therefore, $F$ has a unique fixed point by Banach's contraction principle.

Theorem 7. Let $f: J \times \mathscr{B} \rightarrow \mathbb{R}$ be a continuous function, and let the following assumptions hold.

(H1) There exist $p, q \in C\left(J, \mathscr{R}^{\geq 0}\right)$ such that $|f(t, u)| \leq p(t)+$ $q(t)\|u\|_{\mathscr{B}}$ for each $t \in J, u \in \mathscr{B}$ and and $\left\|I^{\alpha} p\right\|<+\infty$.

(H2) The function $g$ is continuous and completely continuous. For any bounded set $\mathscr{D}$ in $\Omega$, the set $\left\{t \rightarrow g\left(t, y_{t}\right)\right.$ : $y \in \mathscr{D}\}$ is equicontinuous in $C([0, b], \mathbb{R})$. There exist 
positive constants $d_{1}$ and $d_{2}$ such that $|g(t, u)| \leq$ $d_{1}\|u\|_{\mathscr{B}}+d_{2}$ for each $t \in[0, b]$ and $u \in \mathscr{B}$.

If $k_{b} d_{1} \in(0,1)$, then (3) has at least one solution on $(-\infty, b]$, where $k_{b}=\sup \{|k(t)|: t \in[0, b]\}$.

Proof. Consider the operator $P^{*}: \Omega \rightarrow \Omega$ defined by

$$
\begin{aligned}
& P^{*}(y)(t) \\
& \quad= \begin{cases}\mathscr{L}(I) y(t)+I^{\alpha} f\left(t, y_{t}\right)+g\left(t, y_{t}\right), & t \in[0, b], \\
\phi(t), & t \in(-\infty, 0],\end{cases}
\end{aligned}
$$

where

$$
\mathscr{L}(I)=\sum_{k=0}^{n}\left(\begin{array}{c}
-\alpha \\
k
\end{array}\right) \frac{n ! t^{n-k}}{(n-k) !} I^{\alpha-\beta+k}
$$

In analog to Theorem 5 , we consider the operator $F^{*}: C_{0} \rightarrow$ $\mathrm{C}_{0}$ defined by

$$
F^{*} z(t)=\mathscr{L}(I) z(t)+I^{\alpha} f\left(t, \bar{z}_{t}+x_{t}\right)+g\left(t, \bar{z}_{t}+x_{t}\right)
$$

By using ( $\mathrm{H} 2)$ and Theorem 5 , the operator $F^{*}$ is continuous and completely continuous. Now, it is sufficient to show that there exists an open set $U^{*} \subseteq C_{0}$ with $z \neq \lambda F^{*}(z)$ for $\gamma \in(0,1)$ and $z \in \partial U^{*}$.

Let $z \in C_{0}$ and $z=\gamma F^{*}(z)$ for some $\gamma \in(0,1)$. Then, for each $t \in[0, b], z(t)=\gamma\left[g\left(t, \bar{z}_{t}+x_{t}\right)+\mathscr{L}(I) z(t)+I^{\alpha} f\left(t, \bar{z}_{t}+\right.\right.$ $\left.x_{t}\right)$ ]. Hence,

$$
\begin{aligned}
|z(t)| \leq & d_{1}\left\|\bar{z}_{t}+x_{t}\right\|_{\mathscr{B}}+d_{2} \\
& +\sum_{k=0}^{n} \frac{\left|\left(\begin{array}{c}
-\alpha \\
k
\end{array}\right)\right| k ! b^{n-k}}{(n-k) ! \Gamma(\alpha-\beta+k+1)}\|z\|_{b} \\
& +\frac{b^{\alpha_{n}}\|p\|_{\infty}}{\Gamma(\alpha+1)}+\frac{1}{\Gamma(\alpha)} \int_{0}^{t}(t-s)^{\alpha_{n}-1} q(s)\left\|\bar{z}_{s}+x_{s}\right\|_{\mathscr{B}} d s, \\
\leq & d_{1} \delta(t)+d_{2}+\frac{b^{\alpha}\|p\|_{\infty}}{\Gamma(\alpha+1)} \\
& +\frac{1}{\Gamma(\alpha)} \int_{0}^{t}(t-s)^{\alpha-1} q(s) \delta(s) d s \\
& +\sum_{k=0}^{n} \frac{\left|\left(\begin{array}{c}
-\alpha \\
k
\end{array}\right)\right| k ! b^{n-k}}{(n-k) ! \Gamma(\alpha-\beta+k+1)}\|z\|_{b},
\end{aligned}
$$

where $\delta(t)$ is named the in right-hand side of (25) such that $\left\|\bar{z}_{s}-x_{s}\right\| \leq \delta(t)$. Since $0<k_{b} d_{1}<1$, if we let $T^{*}=$ $\sum_{k=0}^{n}\left(\left|\left(\begin{array}{c}-\alpha \\ k\end{array}\right)\right| k ! b^{n-k}\|z\|_{b} k_{b} /(n-k) ! \Gamma(\alpha-\beta+k+1)\right)$, then

$$
\begin{gathered}
\delta(t) \leq k_{b} d_{1} \delta(t)+k_{b} d_{2}+m_{b}\|\phi\|_{\mathscr{B}}+T^{*}+m_{b}\|\phi\|_{\mathscr{B}} \\
+\frac{k_{b} b^{\alpha}\|p\|_{\infty}}{\Gamma(\alpha+1)}+\frac{k_{b}\|q\|_{\infty}}{\Gamma(\alpha)} \int_{0}^{t}(t-s)^{\alpha-1} \delta(s) d s \\
\leq \frac{1}{1-k_{b} d_{1}}\left\{k_{b} d_{2}+m_{b}\|\phi\|_{\mathscr{B}}+T^{*}+m_{b}\|\phi\|_{\mathscr{B}}\right. \\
+\frac{k_{b} b^{\alpha}\|p\|_{\infty}}{\Gamma(\alpha+1)}+\frac{k_{b}\|q\|_{\infty}}{\Gamma(\alpha)} \\
\left.\times \int_{0}^{t}(t-s)^{\alpha-1} \delta(s) d s\right\} .
\end{gathered}
$$

Then, using Lemma 3, there exists a constant $\Delta^{*}$ such that

$$
\begin{aligned}
& \delta(t) \leq k_{b} d_{1} \delta(t)+k_{b} d_{2}+m_{b}\|\phi\|_{\mathscr{B}} \\
&+T^{*}+m_{b}\|\phi\|_{\mathscr{B}}+\frac{k_{b} b^{\alpha}\|p\|_{\infty}}{\Gamma(\alpha+1)} \\
&+\frac{k_{b}\|q\|_{\infty}}{\Gamma(\alpha)} \int_{0}^{t}(t-s)^{\alpha-1} \delta(s) d s \\
& \leq \frac{1}{1-k_{b} d_{1}} \\
& \times\left\{k_{b} d_{2}+m_{b}\|\phi\|_{\mathscr{B}}+T^{*}+m_{b}\|\phi\|_{\mathscr{B}}+\frac{k_{b} b^{\alpha}\|p\|_{\infty}}{\Gamma(\alpha+1)}\right. \\
&\left.\quad+\Delta^{*} \frac{k_{b}\|q\|_{\infty}}{\Gamma(\alpha)} \int_{0}^{t}(t-s)^{\alpha-1} \delta(s) d s\right\},
\end{aligned}
$$

and, therefore, $\|w\|_{\infty} \leq R+R \Delta^{*} k_{b}\left\|q^{*}\right\|_{\infty} / \Gamma(\alpha+1):=L^{*}$, where $\left\|q^{*}\right\|_{\infty}=\|q\|_{\infty} /\left(1-k_{b} d_{1}\right)$ and $R=1 /\left(1-k_{b} d_{1}\right)\left[k_{b} d_{2}+\right.$ $\left.m_{b}\|\phi\|_{\mathscr{B}}+\left(k_{b} b^{\alpha}\|p\|_{\infty}\right) / \Gamma(\alpha+1)+T^{*}\right]$. Then,

$$
\|z\|_{\infty} \leq d_{1} L^{*}+d_{2}+\frac{b^{\alpha}\|p\|_{\infty}}{\Gamma(\alpha+1)}+L\left\|I^{\alpha} q\right\|_{\infty}+T^{*}
$$

and, hence,

$$
\|z\|_{\infty} \leq \frac{d_{1} L^{*}+d_{2}+b^{\alpha}\|p\|_{\infty} / \Gamma(\alpha+1)+L^{*}\left\|I^{\alpha} q\right\|_{\infty}}{1-\|z\|_{\infty} T^{*}}:=M^{*} .
$$

Set $U^{*}=\left\{z \in C_{0}:\|z\|_{b}<M^{*}+1\right\}$. From the choice of $U^{*}$, there is no $z \in \partial U^{*}$ such that $z=\gamma F^{*}(z)$ for $\gamma \in(0,1)$. As a consequence of the nonlinear alternative of the LeraySchauder theorem, we deduce that $F^{*}$ has a fixed-point $z^{*}$ in $U^{*}$, which is a solution of (3).

The unique solution of (3), under some conditions, is studied in the following theorem which is the result of the Banach contraction mapping. 
Theorem 8. Let $f: J \times \mathscr{B} \rightarrow \mathbb{R}$ be a continuous function, and there exist positive constants $l, \mu$, such that

$$
\begin{aligned}
& |f(t, u)-f(t, v)| \leq l\|u-v\|_{\mathscr{B}}, \\
& |g(t, u)-g(t, v)| \leq \mu\|u-v\|_{\mathscr{B}},
\end{aligned}
$$

where $t \in J$ and $u, v \in \mathscr{B}$. Then, (3) with the following conditions has a unique solution in the interval $(-\infty, b]$

$$
0<T+\frac{l k_{b} b^{\alpha}}{\Gamma(\alpha+1)}<1, \quad 0<k_{b} \mu+T+\frac{k_{b} l b^{\alpha}}{\Gamma(\alpha+1)}<1,
$$

such that $T$ is defined in Theorem 6.

Proof. The proof is a similar process Theorem 6.

\section{Conclusions}

In this paper, the existence and the uniqueness of solutions for the nonlinear fractional differential equations with infinite delay comprising standard Riemann-Liouville derivatives have been discussed in the phase space. Leray-Schauder's alternative theorem and the Banach contraction principle were used to prove the obtained results. Further generalizations can be developed to some other class of fractional differential equations such as $\mathscr{L}(D) y(t)=f\left(t, y_{t}\right)$, where $\mathscr{L}(D)=D^{\alpha_{n}}-\sum_{j=1}^{n-1} p_{j}(t) D^{\alpha_{n-j}}, 0<\alpha_{1}<\cdots<\alpha_{n}<$ $1, p_{j}(t)=\sum_{k=0}^{N_{j}} a_{j k} t^{k}$, and $N_{j}$ is nonnegative integer.

\section{Acknowledgments}

The authors would like to thank the referee for helpful comments and suggestions. This paper was funded by King Abdulaziz University, under Grant no. (130-1-1433/HiCi). The authors, therefore, acknowledge technical and financial support of KAU.

\section{References}

[1] A. A. Kilbas, H. M. Srivastava, and J. J. Trujillo, Theory and Applications of Fractional Differential Equations, vol. 204 of North-Holland Mathematics Studies, Elsevier Science B.V., Amsterdam, The Netherlands, 2006.

[2] K. S. Miller and B. Ross, An Introduction to the Fractional Calculus and Fractional Differential Equations, A Wiley-Interscience Publication, John Wiley \& Sons, New York, NY, SUA, 1993.

[3] K. B. Oldham and J. Spanier, The Fractional Calculus, Academic Press, New York, NY, USA, 1974.

[4] I. Podlubny, Fractional Differential Equations, vol. 198 of Mathematics in Science and Engineering, Academic Press, San Diego, Calif, USA, 1999.

[5] S. G. Samko, A. A. Kilbas, and O. I. Marichev, Fractional Integrals and Derivatives, Gordon and Breach Science, Yverdon, Switzerland, 1993.

[6] D. Baleanu, K. Diethelm, E. Scalas, and J. J. Trujillo, Fractional Calculus, vol. 3 of Series on Complexity, Nonlinearity and Chaos, World Scientific, Hackensack, NJ, USA, 2012.
[7] W. G. Glockle and T. F. Nonnenmacher, "A fractional calculus approach of self-similar protein dynamics," Biophysical Journal, vol. 68 , pp. 46-53, 1995.

[8] D. Baleanu, Z. B. G. Günvenc, and J. A. T. Machado, New Trends in Nanotechnology and Fractional Calculus Applications, Springer, New York, NY, USA, 2010.

[9] K. Diethelm and A. D. Freed, "On the solution of fractional order differential equations used in the modeling of viscoplasticity," in Scientific Computing in Chemical Engineering IIComputional Fluid Dynamics, Reaction Engineering and Molecular Properties, F. Keil, W. Mackens, H. Voss, and J. Werther, Eds., pp. 217-224, Springer, Heidelberg, Germany, 1999.

[10] A. Babakhani and V. Daftardar-Gejji, "Existence of positive solutions of nonlinear fractional differential equations," Journal of Mathematical Analysis and Applications, vol. 278, no. 2, pp. 434-442, 2003.

[11] C. Yu and G. Gao, "Existence of fractional differential equations," Journal of Mathematical Analysis and Applications, vol. 310, no. 1, pp. 26-29, 2005.

[12] A. Babakhani and V. Daftardar-Gejji, "Existence of positive solutions for $N$-term non-autonomous fractional differential equations," Positivity, vol. 9, no. 2, pp. 193-206, 2005.

[13] D. Băleanu, O. G. Mustafa, and R. P. Agarwal, "Asymptotically linear solutions for some linear fractional differential equations," Abstract and Applied Analysis, vol. 2010, Article ID 865139, 8 pages, 2010.

[14] A. Cabada and G. Wang, "Positive solutions of nonlinear fractional differential equations with integral boundary value conditions," Journal of Mathematical Analysis and Applications, vol. 389, no. 1, pp. 403-411, 2012.

[15] B. Ahmad, J. J. Nieto, A. Alsaedi, and M. El-Shahed, "A study of nonlinear Langevin equation involving two fractional orders in different intervals," Nonlinear Analysis: Real World Applications, vol. 13, no. 2, pp. 599-606, 2012.

[16] S. Bhalekar, V. Daftardar-Gejji, D. Baleanu, and R. Magin, "Fractional Bloch equation with delay," Computers \& Mathematics with Applications, vol. 61, no. 5, pp. 1355-1365, 2011.

[17] R. P. Agarwal and B. Ahmad, "Existence theory for anti-periodic boundary value problems of fractional differential equations and inclusions," Computers \& Mathematics with Applications, vol. 62, no. 3, pp. 1200-1214, 2011.

[18] B. Ahmad, J. J. Nieto, and A. Alsaedi, "A nonlocal three-point inclusion problem of Langevin equation with two different fractional orders," Advances in Difference Equations, vol. 2012, article 54, 2012.

[19] D. Baleanu and J. J. Trujillo, "On exact solutions of a class of fractional Euler-Lagrange equations," Nonlinear Dynamics, vol. 52, no. 4, pp. 331-335, 2008.

[20] K. Diethelm and N. J. Ford, "Analysis of fractional differential equations," Journal of Mathematical Analysis and Applications, vol. 265, no. 2, pp. 229-248, 2002.

[21] K. Diethelm, "An algorithm for the numerical solution of differential equations of fractional order," Electronic Transactions on Numerical Analysis, vol. 5, pp. 1-6, 1997.

[22] Z. Odibat and S. Momani, "Numerical methods for nonlinear partial differential equations of fractional order," Applied Mathematical Modelling, vol. 32, pp. 28-39, 2008.

[23] K. Diethelm and G. Walz, "Numerical solution of fractional order differential equations by extrapolation," Numerical Algorithms, vol. 16, no. 3-4, pp. 231-253, 1997. 
[24] T. Maraaba, D. Baleanu, and F. Jarad, "Existence and uniqueness theorem for a class of delay differential equations with left and right Caputo fractional derivatives," Journal of Mathematical Physics, vol. 49, no. 8, Article ID 083507, 11 pages, 2008.

[25] H. Ye, Y. Ding, and J. Gao, "The existence of a positive solution of a fractional differential equation with delay," Positivity, vol. 11, pp. 341-350, 2007.

[26] J. K. Hale and J. Kato, "Phase space for retarded equations with infinite delay," Funkcialaj Ekvacioj, vol. 21, no. 1, pp. 11-41, 1978.

[27] A. Granas and J. Dugundji, Fixed Point Theory, Springer Monographs in Mathematics, Springer, New York, NY, USA, 2003.

[28] R. R. Golberg, Methods of Real Analysis, Oxford and IBH Publishing Company, New Delhi, India, 1970.

[29] D. Henry, Geometric Theory of Semilinear Parabolic Partial Differential Equations, Springer, Berlin, Germany, 1989. 


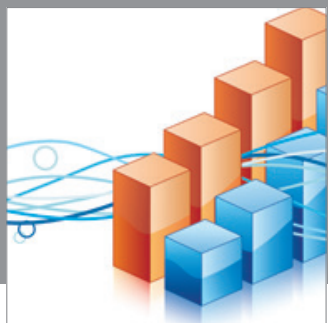

Advances in

Operations Research

mansans

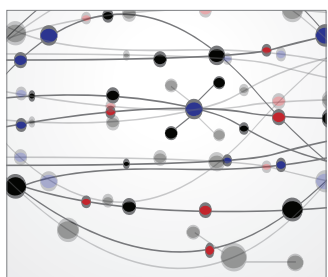

The Scientific World Journal
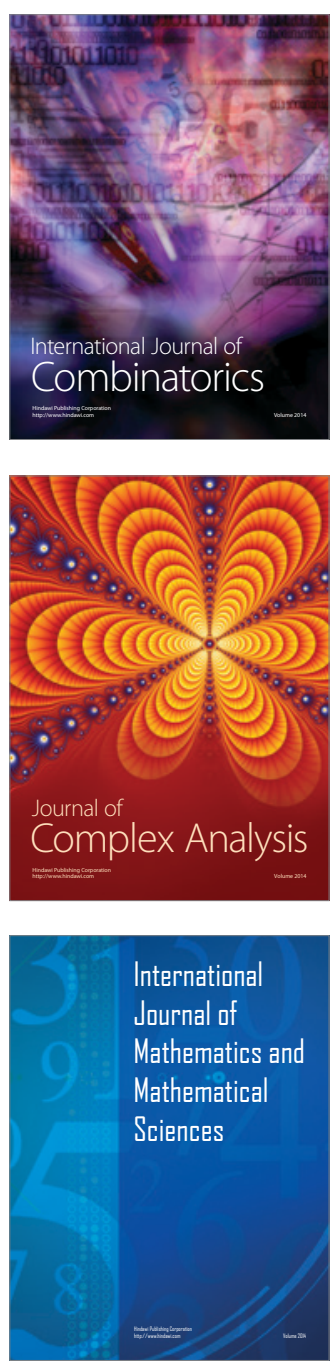
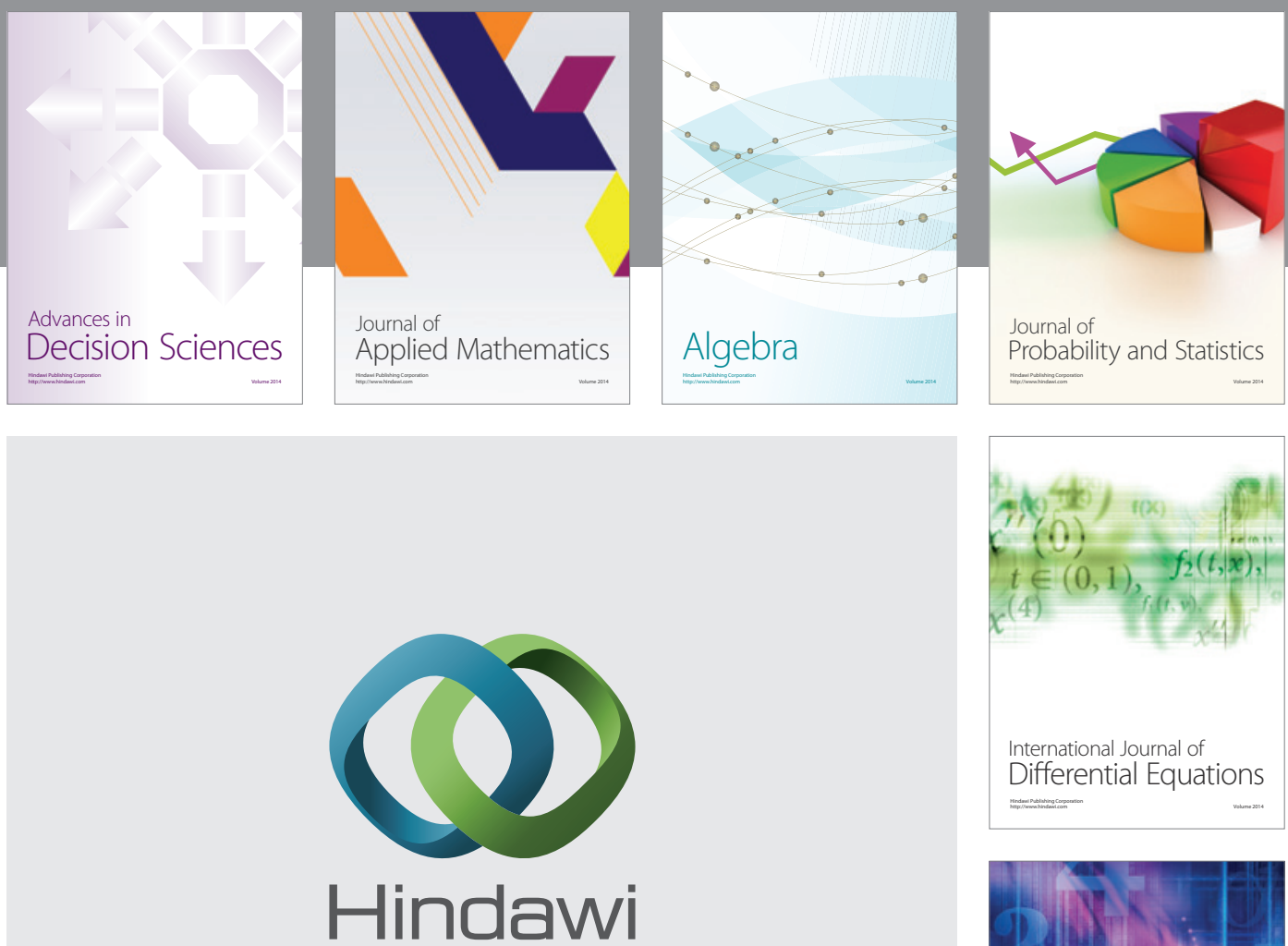

Submit your manuscripts at http://www.hindawi.com
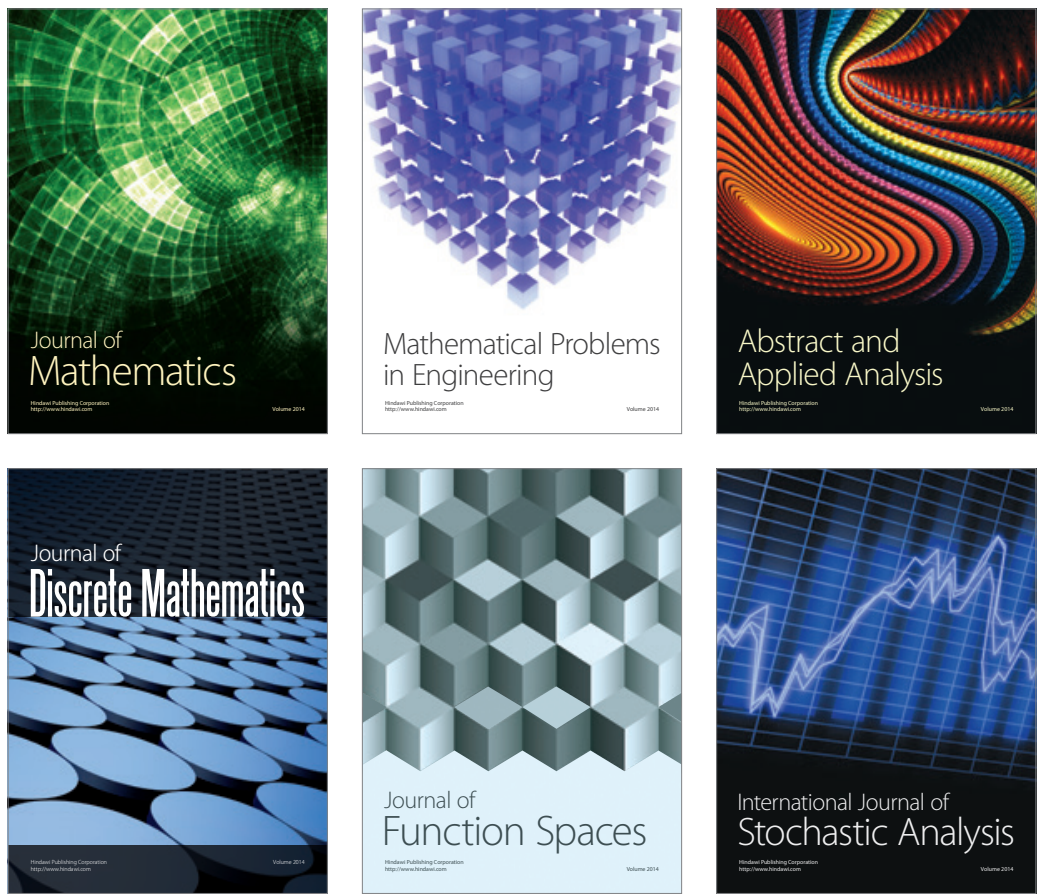

Journal of

Function Spaces

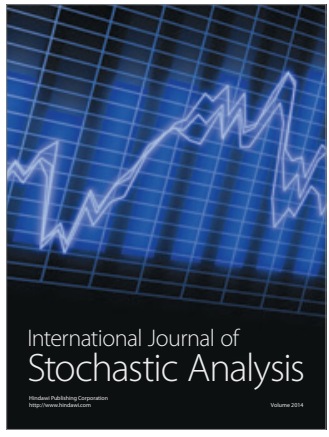

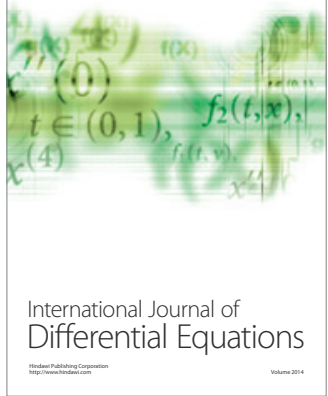
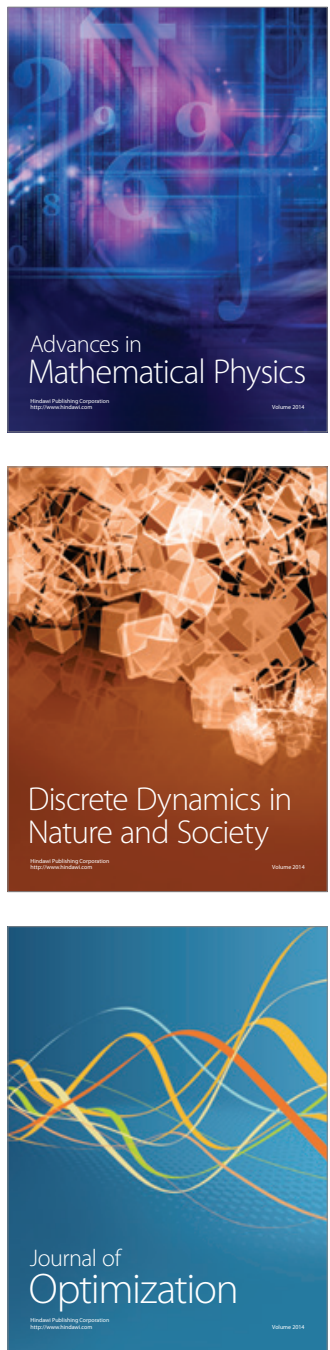